\title{
On Target: Progress in serial crystallography using fixed targets at XFELs and Synchrotrons.
}

\author{
Danny Axford, Darren Sherrell and Robin L Owen. \\ Diamond Light Source, Harwell Oxford, Didcot, OX110DE, UK
}

$X$ ray Free Electron Lasers have driven the development of serial crystallography as a result of each ultra-brief, ultra-brilliant $X$-ray pulse destroying sample immediately after interaction. The challenge of presenting a fresh crystal to each new pulse has been tackled in a number of ways by different researchers. Our solution utilises a high density sample array in conjunction with high-precision translation stages, which enable the efficient use of XFEL beam time with frugal sample consumption. Tens of thousands of protein crystals can be held on a compact silicon wafer array and addressed sequentially at rapid rates. The 124 microfocus beamline at Diamond Light Source has developed a highly effective motion control system enabling adaptable sub-micron positioning at rates of over $100 \mathrm{~Hz}$ using XYZ piezo stages. The sample stage system is portable and equally at home on an XFEL or synchroton beamline. The use of fixed targets opens the door to the pre-screening of sample preparations with methods such as UV-vis spectroscopy, or non-linear imaging. Our motion system is then able to harness this information to enable more efficient use of beamtime and processing resources. Our serial method enables room temperature structure solution at the synchrotron in an extremely efficient manner with a markedly reduced X-ray dose relative to conventional rotation experiments. In addition, by collecting serial data in the same manner at both types of light source it is possible to exploit the typically better characterised nature of incident Xrays and detectors at synchrotrons which can then facilitate the interpretation of XFEL measurements. Latest results from experiments at both XFEL and microfocus synchrotron beamline 124 are presented and discussed. 